\title{
Prevention and Reasons Analysis of Volleyball Training Injury
}

\author{
Dupin Xie , Xingxing Liu
}

Nanchang Institute of Science \&Technology,Nanchang 330108,China

Keywords: Volleyball training; prevention strategy; injury

\begin{abstract}
Athletes often occurrence sport injuries in volleyball training. The main cause of this event is due to the lack of awareness of athletes is not clear. There is some athletes in the injury situation is still not fully recovered with practice, it is very big harm to athletes. Therefore, in order to minimize the damage of volleyball athletes during training events, the occurrence regularity of damage of the athletes, more perfect and injury prevention work for the future to do and put forward some suggestions and opinions. It is of great practical significance to ensure the healthy development of the mobilization.
\end{abstract}

As an important sport of all kinds of schools in our country, volleyball has strong technical skills, and confrontational, it is a skill type sports day net class confrontation, although the two small force will occur physical contact and confrontation, but with a strong psychological quality, physical quality and the technical and tactical requirements in sports, volleyball training course if you do not pay attention to prevention of sports injury, most likely caused by sports injury in the training process. Therefore, it is of great significance for the prevention of sports injury and the improvement of the overall level of volleyball training, to strengthen the prevention and analysis of sports injury in volleyball training.

\section{What volleyball injury is}

The volleyball injury refers to all injury in volleyball, occurrence factors of volleyball injury has multiple, related to the technical movement, training arrangement, training level, psychological quality and other factors, especially because of volleyball technology often is done in the squat state or jump into the air. It is very easy to cause injury of the knee, stomp, waist, shoulders and other parts.

The characteristics of volleyball outstanding, not only requires more height, but also must have the required sensitivity, strength and endurance, which requires high quality cable and psychological quality for the body, according to the investigation and analysis of China's volleyball players and domestic experts and scholars, Chinese male volleyball players set up newly in 2001, with the knee, the waist and ankle part of infants incidence rate is the highest, respectively, 21.5\%, $17 \%$ and $15 \%$, followed by hand, shoulder, wrist and other parts, other parts are relatively low, and many of the chronic injury, while in volleyball training, then to the majority of acute injury in chronic strain is also more and more, and with the movement of life the more damage growth.

\section{Cause analysis of athlete injury}

As a kind of anti competitive sports event, the volleyball game. Compared to other sports injury rate is low, the players in training, there will not be too much physical contact, etc., but the athlete's injury can not be completely avoided. The following are the main reasons for the injury of athletes. 
Volleyball sports on students' physical quality and psychological quality requirements, the quality of these two forces, if small, easily lead to sports injury in volleyball training. Quality requirements of volleyball jumping ability is the most important for the athletes, especially in the new situation of volleyball technology change rapidly under the net strength is also more and more high, the attack mode is also more and more, so the ability to bounce and bounce technology requirements are increasingly high, but in the volleyball training process, students and the students' physical quality and psychological quality has great difference, because in the training process can not take the classification training, so it is easy to make some physical and psychological qualities of small good student movement, there is a part of students' physical quality and psychological quality is not strong, so in the process of learning volleyball movement skill is not in place, due to technical errors will lead to sports injury.

Athletes lack of awareness of injury prevention. In volleyball training, many coaches pay more attention to the explanation of tactics and basic technology, but not too much attention to the safety consciousness of athletes. Because of the complexity of volleyball, in the process of training, often need to jump or move. Especially for the athletes spiking practice, if its own lack of awareness of the damage is likely to make the presence of any rolling and volleyball spiking athletes tread, caused by knee or ankle injury. Therefore, coaches and athletes themselves need to strengthen the awareness of injury prevention, so as to be able to nip in the bud.

Lack of enough prepare before training. In a lot of professional volleyball training activities, the coaches do not attach enough importance to the preparation activities, resulting in the injury of athletes in sports. This kind of volleyball training is contrary to the basic law of human body movement and the law of physical education. In volleyball training, shoulder heavy burden, if not to be able to do some preparation activities of the joint after high intensity training and service is very likely to cause the spike, joint injury. Therefore, no matter what kind of sports training activities, must be prepared before the training as a prerequisite, if not combined with the characteristics of the preparation of sports events before the training, it will undoubtedly cause injury.

Training level is not enough. Action is not standardized. Because of the complexity of the technique of volleyball, there are different structural changes for each technique. In the long term development of the sport, its action is in line with the principles of human physiology. Therefore, if the players can correctly grasp the various techniques of volleyball, in general, it will not lead to the occurrence of sports injuries. In volleyball teaching, a large part of the reason for the injury of athletes is that they do not have a good grasp of volleyball skills. For example, in the initial stage of the volleyball pass technique learning, if the hand when the ball is not very easy to cause finger joint injury.

\section{Prevention strategies of injury in volleyball training}

Cultivate the athletes' safety consciousness. In the beginning of the formal training, should convey knowledge of safety education to the athletes; let their own to enhance their safety awareness, so that it can be possible on the train from the thought of the damage and harm to have a more clear understanding. At the same time, the cultivation of safety awareness should be run through the whole training process. Enable students to grasp the scientific method of prevention of injury in the specific practical training, so as to make it as far as possible to reduce the damage to the minimum.

Preparation activities should be reasonable and reasonable, reasonable training. In every time volleyball training, the training content or training focus is different, that before the training activities should also be scientific and reasonable arrangement, the only way to make the muscles and joints in athletes during training activities effectively. This targeted activity is conducive to 
promoting the body's central nervous system excitement, while increasing their muscle strength; improve the flexibility of each joint. As a preparation activity can be very effective to achieve the purpose of preventing athlete injury.

According to the data of sports training, it is reasonable to arrange the amount of exercise, which can not only improve the athletic performance, but also protect the athletes. Because of excessive exercise will exceed the sport organs and organs bear ability, in this case, easy to cause the athlete's physical and mental fatigue, and if so, the ability of athletes will be significantly decreased. Specific manifestations are: inattention, slow reaction, reduced alertness, which has become an important cause of sports injury athletes. Therefore, exercise arrangement should be reasonable, given enough time, it differs from man to man. Athletes' body information and sleep time to ensure the good condition.

Improve the level of volleyball movement. Athletes because they do not have a good grasp of the technical movement of volleyball and sports injuries caused by the phenomenon abound. In the training process, in addition to the coach's careful explanation, but also need to be able to strict standards to study athletes to do the basic technical specifications. In the training of athletes, we should adhere to the principle of gradual and orderly, so that students continue to strengthen the training of basic skills, as a basis for the gradual learning of new technical action. Only when the athletes are skilled and skilled in the technical movements, can they reduce the incidence of sports injuries after training and competition.

Strengthen the psychological training of athletes. A lot of players who want to compete with the human race, their psychological quality is weak will also learn to play in the playground to be hampered or even the situation of sports injury. A lot of players are tactical, physical and so on. Evenly matched, but in the face of the game, the psychological quality is not strong enough to highlight. Therefore, in the training of athletes, we should pay enough attention to the psychological quality training. Let the athletes in the game, regardless of what kind of ability to adjust the mentality, only in this way can play a normal play, to avoid unnecessary mistakes. First of all, the psychological education for athletes should give priority to prevention, action and action in the new difficult to have enough attention to more dangerous action key to master, secondly, injuries will athletes body damage, and also to the athletes' psychological cause two damage, injured athletes if long-term depression, anger, the frustration and pain, the time is longer, the longer the recovery, the possibility of emotional unrest will increase more. Therefore, we should pay attention to the recovery of athletes' recovery period on the basis of psychological prevention.

Arrange trainings reasonable. Volleyball training step by step, the only way to improve the effectiveness of training and to prevent sports injury, and in the training process must be reasonable arrangement of the training content, to strengthen the training of volleyball activities, especially to focus on improving the effect of preparatory activities, reasonable arrangements for time, place and content of activities, especially the special content according to the special volleyball training preparation activities, can do sports injury prevention work in the process of. Due to improper technique is often the cause of sports injury, the correctness and feasibility of the reasonable arrangement of the training is to further strengthen the technical training, efforts to strengthen the protection of training, so in the beginning of training is to make the students understand the possible action and technical essentials, to master the technical movements and technical specifications should be correct, let the students master the technology, but also enable students to obtain the corresponding muscle strength training. For example, in the training of students take off technique action at the same time, must jump and buffer action closely together, this technique is easy to be ignored, and will closely combine can effectively reduce and prevent the occurrence of sports 
injury.

\section{Conclusion}

To sum up, the main cause of injury for volleyball players. Lack of activity, safety awareness is not high; the movement is not accurate, etc... To solve these problems, the author puts forward very targeted solutions that enhance safety awareness of athletes training, good athletes before training activities, improve the accuracy of player skill, strengthen the athletes' psychological quality etc.. Because of personal vision and ability is limited, in this paper, the athletes injury and prevention of the proposed strategy is not comprehensive enough, in the actual training, there are a lot of athletes may lead to injury, need in practice, constantly explore and summarize more reasons, in order to provide more meaningful recommendations for the prevention of motion injury of athletes.

\section{References}

[1] Liu Y. Prevention and reason analysis of volleyball training injury[J]. Contemporary Sports Technology, 2014.

[2] Liu B G. Survey and Analysis of sports injuries in university extracurricular volleyball training[J]. Journal of Shandong Institute of Physical Education \& Sports, 2008.

[3] Sun X. Investigation and Analysis of Injury and Prevention in Volleyball Sports[J]. Journal of Kunming Teachers College, 1999, 42(10):942-951.

[4] Mulatibieke H, Alimujiang Y T, Aniwar M. The Mechanism and Prevention of the Common Athletic Injuries in Volleyball Teaching and Training[M]// Future Computer, Communication, Control and Automation. Springer Berlin Heidelberg, 2011:221-228.

[5] Jr B W, Kacmar L. Common injuries in volleyball. Mechanisms of injury, prevention and rehabilitation[J]. Sports Medicine, 1997, 24(1):65.

[6]Monteleone G, Tiloca A, Sorge R, et al. The influence of plantar vault shape on ankle sprain epidemiology in volleyball: preliminary results[J]. 2012. 